\title{
Dissociation between the impact of evidence on eye movement target choice and confidence judgements
}

Article

Accepted Version

McSorley, E., Lyne, C. L. and McCloy, R. (2014) Dissociation between the impact of evidence on eye movement target choice and confidence judgements. Experimental Brain Research, 232 (6). pp. 1927-1940. ISSN 1432-1106 doi: https://doi.org/10.1007/s00221-014-3884-2 Available at https://centaur.reading.ac.uk/36034/

It is advisable to refer to the publisher's version if you intend to cite from the work. See Guidance on citing.

To link to this article DOI: http://dx.doi.org/10.1007/s00221-014-3884-2

Publisher: Springer

All outputs in CentAUR are protected by Intellectual Property Rights law, including copyright law. Copyright and IPR is retained by the creators or other copyright holders. Terms and conditions for use of this material are defined in the End User Agreement.

www.reading.ac.uk/centaur 
Central Archive at the University of Reading

Reading's research outputs online 
Dissociation between the impact of evidence on eye movement target choice and confidence judgements

Eugene McSorley*

Clare Lyne

Rachel McCloy

Department of Psychology, University of Reading, Reading, Berkshire, RG6 6AL, UK (corresponding author - e.mcsorley@reading.ac.uk) 


\section{Abstract}

It has been suggested that the evidence used to support a decision to move our eyes and the confidence we have in that decision are derived from a common source. Alternatively, confidence may be based on further post-decisional processes. In three experiments we examined this. In Experiment 1, participants chose between two targets on the basis of varying levels of evidence (i.e., the direction of motion coherence in a Random-Dot-Kinematogram). They indicated this choice by making a saccade to one of two targets and then indicated their confidence. Saccade trajectory deviation was taken as a measure of the inhibition of the non-selected target. We found that as evidence increased so did confidence and deviations of saccade trajectory away from the non-selected target. However, a correlational analysis suggested they were not related. In Experiment 2 an option to opt-out of the choice was offered on some trials if choice proved too difficult. In this way we isolated trials on which confidence in target selection was high (i.e., when the option to opt-out was available but not taken). Again saccade trajectory deviations were found not to differ in relation to confidence. In Experiment 3 we directly manipulated confidence, such that participants had high or low task confidence. They showed no differences in saccade trajectory deviations. These results support post-decisional accounts of confidence: evidence supporting the decision to move the eyes is reflected in saccade control, but the confidence that we have in that choice is subject to further post-decisional processes.

Keywords: Saccades Decision Making Confidence 


\section{Introduction}

In our daily lives, we are continually making decisions about where to look next. These on-going perceptual decisions result in eye movements that occur 3-4 times a second. This decision-making process is often viewed as a competition between potential target objects in the visual scene to become the location for the next saccade (Findlay and Walker 1999). In order to make a saccade to a chosen target, other stimuli need to be inhibited by the saccadic system. This is reflected in changes in the control of the eye movement. Changes are seen in the dynamics and metrics of the eye such as the latency of response (reaction time), the trajectory (its path of travel) and the landing position, and have been interpreted as indicative of the underlying competitive process which culminate in a perceptual decision (McSorley et al. 2009; McSorley and McCloy 2009; McSorley et al. 2012; Meeter et al. 2010; Tipper et al. 2001; Van der Stigchel et al. 2006). However the majority of experiments examining saccadic eye movement control in target selection have largely removed the element of target choice as the target identity is known prior to the experiment. Hence the decision becomes not about target choice so much as target localization. Thus they do not show whether the processes involved in making the decision underlying target choice influence saccade control.

Taking the lead from a large body of work examining the neural activity involved in perceptual decision making, specifically in situations of eye movement target choice in which target identity is supported by different levels of evidence (Britten et al. 1996; Gold and Shadlen 2001; Gold and Shadlen 2000; Horwitz and Newsome 2001; Kim and Shadlen 1999; Roitman and Shadlen 2002; Shadlen and Newsome 
2001), McSorley and McCloy (2009) carried out an experiment in order to examine how, or if, the decision making element of saccade choice is reflected in the dynamics and metrics of the eye movement. They varied the amount of evidence (the motion direction indicated by different levels of motion coherence in a random dot kinematogram - RDK) indicating the saccade target and found that the average saccade latency decreased with increasing evidence, whereas the number of correct responses increased. Importantly they also found that the trajectories and landing positions of correct saccades deviated away from the non-selected target, and on both measures this deviation increased with higher levels of supporting evidence. This suggests that the level of evidence is directly reflected in the programming of the saccade. McSorley and McCloy suggest that there is a quicker development of inhibition for higher levels of evidence, leading to an increased deviation away from the inhibited non-selected target. This was taken to reflect underlying neural activity associated with accumulation of evidence and the competition between targets. These results suggest that saccade deviations and landing positions can represent the activation state of neurons involved in the decision making process.

Accompanying decisions is a sense of confidence (Yeung and Summerfield 2012), and it is generally assumed that people form beliefs about the likelihood that they will get the decision right (Harvey 1997). There have been suggestions (Audley 1964; Link 1992; Petrusic and Baranski 2003; Vickers 1979) that the same mechanisms that underlie the decision making process also underlie confidence judgements. There is a class of models that assumes that confidence is based on the quality and quantity of the evidence available for a choice (decisional locus models). The quality of evidence is represented by how quickly evidence is accumulated in favour of one 
alternative, and the quantity is represented by a bound that the accumulation needs to reach in order for a decision to be made. Decisional locus models state that confidence in the decision can be read out directly from the accumulated evidence that supports the choice at the time the decision was made (e.g., Kepecs et al. 2008; Kiani and Shadlen 2009). In support of this, Kiani and Shadlen (2009) have recently shown that choice confidence using an RDK task can be related to the variable discharge of LIP neurons, such that intermediate levels of discharge (where levels of activation associated with one choice was not appreciably greater than that supporting the other) were found on trials in which non-human primates elected to opt-out of saccade target choice to take a small but certain reward. This suggests that degree of certainty in the choice made and not just the choice itself could be reflected in the competitive processes which underlie the control of the saccadic eye movements: i.e., deviations in the metrics and dynamics of the saccade may not just reflect the choice but also the confidence in that choice.

However, there is conflicting evidence that suggests that a sense of confidence is not purely based on the same information that led to the choice itself, but rather results from additional post-decisional processing (Juslin and Olsson 1997; Vickers 1979; Yeung and Summerfield 2012). For example, Pleskac and Busemeyer (2010) present a model that accounts for distributions of choice, reaction times and confidence ratings in a two-stage model. The first stage consists of sequential sampling of evidence in a random walk process (Laming 1968; Link and Heath 1975; Ratcliff 1978; Stone 1960) that gradually drifts in favour of one alternative over the other, and eventually reaches a pre-set level at which a choice is made. Both choice and decision times are a compromise between the quality and the quantity of the 
evidence. In a second-stage, confidence ratings are made on the basis of postdecisional processing. This is conceived as being a continuation of evidence accumulation that took place in the first "decision" stage. For example, this might take the form of a fixed temporal window over which further evidence accumulation takes place. The state of the evidence supporting either choice at this point forms the basis of the confidence rating (e.g., Pleskac \& Busemeyer, 2010). The authors do not postulate how the evidence is gathered, allowing that it could be a measure of the match between a test item and a memory (Ratcliff 1978) or even the difference in activation between two pools of neurons (Gold and Shadlen 2002; Gold and Shadlen 2001).

In this paper, we aim to examine whether confidence in a choice is derived from the same neural firing rates as those responsible for eye movement control when executing a perceptual choice or whether confidence and eye movement control maybe based on the same source but are ultimately separable in some sense (e.g., through post-decisional processing). To this end a series of three experiments were carried out in which observers were asked to make a motion discrimination decision on the basis of varying amounts of supporting evidence in an RDK. In the first, as well as making a saccade to a target on the basis of the motion inherent in the motion coherence stimuli, participants were also required to make a confidence judgement. If confidence in a choice were derived from the same neural firing rates as those responsible for eye movement control then different amounts of supporting evidence would be expected to have a similar effect on choice confidence as they do on saccade metrics and dynamics. In the second, we introduced an opt-out visual target that could be selected if the participants were unsure of the motion direction. 
Following Kiani \& Shadlen (2009), we reasoned that correctly directed saccades made on trials in which an opt-out target was available but was not selected would be associated with greater confidence. We would therefore expect to see greater trajectory deviation on those trials relative to those in which no opt-out target was presented. In the third, we directly examined whether confidence could impact on saccade control by manipulating participants' confidence independent of actual performance. If it is the case that confidence judgement and saccade control are derived from the same underlying activity then it should be the case that different levels of pre-existing confidence may change this underlying activity. Thus being more confident in target choice more generally may reduce saccade latencies and increase trajectory deviations away from the non-selected target by increasing the extent of inhibition of the target or the speed by which that inhibition develops. In order to examine the impact of confidence on saccade deviation in target choice, we provided manipulated feedback to two groups of participants regarding their performance on the same RDK task as in Experiment 1. This instilled differing levels of confidence between the two groups independently of the external evidence. In a subsequent block of trials, with unmodified feedback appropriate to their choices, we examined whether the differences in confidence felt by the two groups impacted on the same underlying activity from which saccade trajectory control is derived or whether saccade trajectory reflected the evidence supporting target selection to the same extent regardless of confidence.

To pre-empt our findings somewhat, we find that choice performance and the associated confidence increased as the evidence supporting the choice increased, but there was little to no relationship between confidence in the choice and the eye 
movement deviations. This suggests that while both eye movement deviations and confidence reflect the perceptual evidence supporting the choice they are to some extent independent of each other.

\section{Experiment 1}

In this first experiment, the motion coherence of an RDK was manipulated in order to provide differing levels of evidence to support target choice. As well as making a saccade to a target based on the motion of the dots, participants were also required to make a confidence judgement. We aimed to establish whether the level of evidence had the same impact on the saccade metrics and the reported confidence. McSorley and McCloy (2009) have shown that the evidence supporting a choice impacts on the saccade metrics, causing a deviation of the movement away from the non-selected target. The authors attribute this to the increased evidence for one target over another resulting in the activation for the non-selected target being inhibited more quickly. This area of inhibition then impacts on the trajectory and landing position of the saccade, leading to a deviation away from the non-selected target. In line with this we would expect to see confidence in target selection also increase with evidence supporting a choice. If these are derived from common mechanisms then the amount of evidence present in the RDK should be expected to similarly affect both the saccade metrics and the reported confidence in the decision. 


\section{Method}

\section{Observers}

20 observers participated in the experiment, 19 of which were naïve undergraduates, and one experimenter (CL). Participants were aged between 18 and 25 years old. All had normal, or corrected to normal eyesight. Local ethical approval was obtained, and the study was conducted in accordance with the standards described in the 1964 Declaration of Helsinki.

\section{Apparatus}

Participants' eye movements (left eye only) were recorded using an Eyelink II, which is a head mounted eye tracker with a $500 \mathrm{~Hz}$ sampling rate. Participants placed their chin on a rest, which constrained any head movements and ensured the viewing distance remained at one metre. Before the experiment began, the eye tracker was calibrated using a 9 point grid, and then validated using a different grid. This was only accepted and the participant allowed to start the experiment when there was an average difference of less than 0.5 degrees between the actual eye position and that predicted from the calibration and the validation. Stimuli were presented on a 21" colour monitor that had a refresh rate of $75 \mathrm{~Hz}$.

A total of 200 visual analogue scales were used, in order to obtain a confidence rating for each decision that a participant made. The scales were presented on paper as $12 \mathrm{~cm}$ lines (10 per page), and labelled 'Low Confidence' on the left end and 'High Confidence' on the right. For each choice, participants were instructed to make a pencil mark anywhere along the scale, representing how confident they were in the decision they had just made. 


\section{Stimuli}

The stimuli consisted of RDKs, which were presented centrally within a circular aperture that measured five degrees of visual angle. The screen was black, while the random dots and targets were white. The motion coherence levels used were 4, 8, 16,32 and $64 \%$, and there were forty trials for each motion coherence level. The coherent dots, which travelled in one of two directions, had a $100 \%$ lifetime whereas the other random noise dots were re-plotted in random locations. The random dot motion was created by spatially relocating a certain amount of the dots, which gave the impression of speed of 6.7 degrees per second along the target axis of 22.5 degrees on either side of the vertical meridian. The dot density of the display was 16.7 dots/deg squared/second. The two targets, placed 8 deg from fixation along the direction axes of the RDKs, were white annuli created by overlaying a smaller black circle (0.5deg in diameter) on a larger white circle ( 1 deg in diameter). A schematic of the task sequence is represented in Figure 1.

\section{FIGURE 1 HERE}

\section{Procedure}

Participants were first familiarized with the stimuli and the task, and were encouraged to carry out as many practice trials as they felt was necessary to become comfortable with the task and what they had to do. Participants were instructed to move their eyes to one of the targets based on the motion they saw in the RDK stimulus, and were told to guess if they were uncertain as to the correct answer. When participants were ready to start the trial, they fixated on a small white annulus and pressed a button that both initiated the trial and corrected for any small 
changes in the position of the participant that may be brought about by looking down at the paper on which they marked their confidence (using a drift correct procedure involving the head camera on the eye tracker and markers attached to the monitor integral to the Eyelink II system). A centrally placed fixation cross was then presented for $800-1200 \mathrm{~ms}$, after which the motion coherence stimulus and the two targets appeared. The motion coherence stimulus appeared at a fixed duration of $125 \mathrm{~ms}$ across all trials while the targets were presented for one second. The participant then indicated their decision as to the direction of motion they perceived in the motion coherence stimulus by making a saccade to one of the two targets. After they had done this, they recorded how confident they were in their decision by drawing a mark on a visual analogue scale. Participants completed two blocks of 100 trials, so 200 trials in total. With practice runs, this took about 45 minutes in total.

\section{Data analysis}

The eye tracking software includes a parser that was used to identify the start and ends of saccades using a 22 degree per second velocity and 8,000 degrees per second squared criteria (SR research Ltd). Further analysis of saccade trajectory was accomplished by using software developed in Matlab (Mathworks Inc.) The first saccades in each trial were used and from these the saccade latency, saccade trajectory and overall direction were calculated. Saccade latency was defined as the amount of time between the presentation of the experimental display and the initiation of the saccade. A correct landing position was defined as an eye movement that landed within a 45 degree window centred on the correct target (see Figure 1 upper right panel for example saccade traces and details on saccade analysis). We examined the deviation of landing position in terms of how far away from the centre 
of the target the eyes landed in angular degrees but this was not found to vary with increasing motion coherence. This was the case in all three experiments reported here. For this reason, and for the sake of brevity, landing position deviations are not reported. ${ }^{1}$

The maximum trajectory deviation of each saccade relative to the direct path between fixation and landing position was determined (see Ludwig and Gilchrist 2002). This was calculated by fitting a second-order polynomial to the saccade trajectory and finding the maximum point of angular deviation from the straight line that joined the saccade start position to its end position.

Saccades were excluded from the analysis as being anticipatory if they were less than $100 \mathrm{~ms}$, and as not responding to the stimuli if they were over $900 \mathrm{~ms}$. This led to 27 trials being excluded.

Confidence ratings were calculated as a measurement in $\mathrm{cm}$, with the low confidence end starting at zero, and increasing in number to a maximum of $12 \mathrm{~cm}$ as the responses became nearer the high confidence end. These responses were then

\footnotetext{
${ }^{1}$ In contrast to McSorley \& McCloy (2009), we did not find an effect of choice and level of evidence on saccade landing position deviation. They showed similar displays to those employed here and used the same task but found, similarly to saccade trajectory deviation, that landing positions were deviated away from the non-selected target and that this deviation increased as the amount of evidence supporting target choice also increased. There are several possible reasons why this may have been case, the most likely being that the targets are relatively far apart (45 degrees) and saccades are generally only consistently affected by competing stimuli within about 30 degrees of the target (Findlay and Brown 2006; McSorley and Findlay 2003; McSorley et al. 2009; Ottes et al. 1985; Walker et al. 1997). This is especially so at the relatively longer latencies elicited in these choice tasks. In contrast, Saccade trajectories continue to be affected by distracting stimuli at distances of up 120 deg from the target (McSorley et al. 2009). We would suggest from this that the online control of saccade trajectory is much more sensitive to the presence of the other competing stimuli than is the final saccade landing position.
} 
normalised to take into account each individual participant's maximum and minimum confidence level, and each converted into a percentage of the participant's maximum rating. Normalising the confidence ratings did not alter the results.

\section{Results}

The results from the 4 different performance measures are shown in Figure 2. As motion coherence increased so the task became easier: the number of correct responses $(a)$ increased $(F(4,76)=15.762, p<0.001)$, while the saccade latency $(b)$ to make that choice decreased $(F(4,76)=2.690, p<0.037)$. As would be expected the confidence (c) in motion direction choice increased steadily with increasing motion coherence $(F(4,76)=14.171, p<0.001)$. Figure $2 d$ shows that trajectories of saccades tended to deviate away from the non-selected target, and that this increased with motion coherence. While a one way ANOVA found that this was not significant $(F(4,76)=1.184, p>0.05)$, a planned trend analysis shows a significant linear relationship in the data showing that saccade trajectory deviations away from the non-selected target increased in magnitude as motion coherence increased $(F(1,19)=4.662, p=0.044)$. The $4 \%$ coherence level can be considered as a baseline as there is little to no perceived supporting evidence for target choice and this is when participants are clearly guessing. Thus saccade trajectory deviation in this condition shows the natural saccade curvature elicited in target choice in the absence of evidence and can be suggested to purely reflect the inhibition of the nonselected target. In these terms, the trend analysis above can be interpreted as showing the impact on trajectory deviations away from this underlying natural 
curvature (This is also the case in Experiment 3 which shows the same linear relation).

\section{FIGURE 2 HERE}

Confidence ratings and trajectory deviation away from the non-selected target both increase in line with motion coherence and it would be expected that the two measures would correlate if they emanated from the same neural signal that represents the level of evidence accumulated for the target choice. To examine this a correlation analysis between the two measures was carried out. First, this was carried out (separately for each participant) between the confidence ratings and trajectory deviations and did not take the level of motion coherence into account. This analysis found no significant relationship (average Pearson's $r=-0.0135$, standard deviation $=0.14368$, with a range from -0.27 to 0.43 ). These correlation values were also found not to be significantly different from zero $(\mathrm{t}(19)=0.810$, $\mathrm{p}=0.428)$. This was broken down in a further set of correlations for each motion coherence level and for each participant. The only significant correlation between confidence ratings and trajectory deviation was found to be at the $16 \%$ level (Motion Coherence levels followed by average Pearson's r: $4 \% 0.064 ; 8 \% 0.161 ; 16 \% 0.277$, $p<0.05 ; 32 \%-0.048 ; 64 \% 0.013$, all other $p ' s>0.05$ ). This analysis suggests that while saccade trajectory deviations and confidence reflect the evidence supporting the choice they must be ultimately derived from different sources perhaps involving different mechanisms or additional processing after the target choice is made. 


\section{Discussion}

This experiment investigated whether increasing the amount of evidence available to base a decision on led to a similar increase in both confidence ratings and trajectory deviation away from the non-selected target. It was found that both confidence levels and saccade trajectory deviation away from the non-selected target significantly increase as motion coherence levels increase. While this could be taken to suggest that saccade trajectory deviation and decision confidence are derived from common mechanisms, this conclusion is not supported by the correlation analysis between confidence ratings and trajectory deviation showing no significant relationship. Thus they may be dissociable at some level, which would be consistent with a postdecisional account of confidence.

This result seems to run counter to findings from two studies which have found that neurons which are involved in the formation of a decision also encode the confidence in that decision (Kiani and Shadlen 2009; Kepecs et al. 2008). Kiani and Shadlen (2009) used the RDK task and recorded from individual neurons in area LIP to investigate whether confidence was encoded in the decision made. The task was similar to the one employed in Experiment 1 here. A target was selected with a saccade depending on the perceived direction motion inherent in an RDK stimulus, which, if correct was rewarded. Kiani and Shadlen also introduced a third 'opt-out' visual target on half of the trials, which, when selected, led to a smaller reward than a correct directional target but was always given. This allowed the non-human primate the choice to opt-out of the decision when they were unsure of the answer. As with previous single neuron recording studies, it was found that as motion coherence increased, the level of firing in the neuron coding for the selected target increased rapidly and to a higher level. When the opt-out target was ignored, the 
choice accuracy was better than on trials where there was no opt-out target option. This implies a decision to opt-out is not just based on stimulus difficulty but also on a sense of uncertainty. Indeed, neuronal activity in LIP only reached intermediate levels when the opt-out clause was selected compared to when a directional target was chosen. This suggests that evidence supporting the choice of one target over the other was insufficient so the opt-out was exercised, thus the confidence in the decision can be said to be reflected in the neuronal activity in LIP.

These findings would suggest that, in an experiment such as ours, if participants were given an option to opt-out but did not choose to take it then saccade trajectory deviations should be greater than when this opt-out option was not present i.e., there should be stronger inhibition of the non-selected target when participants choose to ignore the opt-out target option because they should be more certain of their choice than when no opt-out option is given. On the other hand, if saccade trajectory deviations reflect the evidence supporting the decision, and are not influenced by the confidence in that decision, then there should be no difference between the magnitude of the deviations whether the opt-out option was present or not. This was examined in Experiment 2.

\section{Experiment 2}

\section{Method}

\section{Observers}

20 observers participated in the experiment, all of whom were naïve undergraduates. Participants were aged between 18 and 26 years old. All had normal, or corrected to 
normal eyesight. Local ethical approval was obtained, and the study was conducted in accordance with the standards described in the 1964 Declaration of Helsinki.

\section{Apparatus}

Apparatus used was the same as Experiment 1.

\section{Stimuli}

The stimuli consisted of a random dot kinematogram, as in Experiment 1. The motion coherence levels used were $15,30,45,60,75 \%$, with a planned forty trials per motion coherence level. These motion coherence levels changed from Experiment 1 as we required a greater range of levels. However, a programming error led to there being fifty trials for the $45 \%$ level, and thirty trials for the $60 \%$ level. This is unlikely to have adversely affected the results and there were sufficient trials for each motion coherence level. See Experiment 1 for stimuli details. The only difference from Experiment 1 was the addition of the opt-out target that was a red circle of 0.5 degrees in diameter, placed eight degrees below fixation on the vertical axis. This appeared on $50 \%$ of the trials.

\section{Procedure}

The procedure is similar to Experiment 1, except that participants were not required to rate their confidence after their choice. Rather they were told to saccade to the opt-out target if they were uncertain as to the correct answer, instead of one of the directional targets. Due to participants not recording their confidence after each trial, 
the drift correct procedure, carried out every trial in Experiment 1, occurred every twenty trials. There were 200 trials in total, which took participants approximately 40 minutes to complete, with practice and set-up.

\section{Data analysis}

Saccade latency and its trajectory were calculated as in Experiment 1. Responses were again categorised as correct if they landed within a 45 degree window centred on the correct target. Opt-out target responses were defined as those that travelled in a downward direction. Saccades were excluded from the analysis as being anticipatory if they were less than $100 \mathrm{~ms}$, and as not responding to the stimuli if they were over $900 \mathrm{~ms}$. This led to 37 saccades being excluded.

\section{Results}

Performance Measures and saccade trajectory deviations

As motion coherence increased, as with Experiment 1, the overall number of correct choices increased $(F(4,72)=57.372, p<0.001$; Figure 3a), the amount of opt-out choices decreased $(F(4,72)=11.822, p<0.001$; Figure $3 b)$, and the overall saccade latency decreased $(F(4,72)=15.523, p<0.001$; Figure $3 c)$. Figure $3 a$ shows that performance was generally better when the opt-out target was not present. A two way ANOVA was carried out with motion coherence levels and whether the opt-out target was present as factors. There was found to be significantly higher levels of performance when the opt-out target was not present $(F(1,18)=8.319, p=0.010)$ and the percentage of correct responses significantly increased as the levels of 
motion coherence also increased $(F(4,72)=57.535, p<0.001)$. Differences across motion coherence depending upon the presence of the opt-out option proved not to be significant (interaction: $F(4,72)=0.964, p=0.433$ ).

The same analysis of saccade latency (Figure 3b) showed the same pattern: there was a significant increase in saccade latency when the opt-out target was present over when it was not present $(F(1,18)=32.059, p<0.001)$; an increase in motion coherence led to a significant decrease in saccade latency; $(F(4,72)=18.463$, $p<0.001$, with a significant linear contrast; $(F(1,18)=32.059, p<0.001)$; and no significant (although trending) interaction $(F(4,72)=2.237, p=.061)$.

It is important to note that while there are not many trials on which participants elected to opt-out of the choice this was sufficient to produce a behavioural impact. All participants (except 1 ) chose to opt-out of making a decision at least once, with $50 \%$ of them electing the opt-out target over 10 times. Overall the opt-out target was selected 216 times and its presence can be seen to be having a behavioural impact on choice performance and the latency of response. Performance was found to differ when an opt-out target was present compared with when it was not present (See Figure 3a). Further analysis of saccade latency showed longer latency responses on trials in which the opt-out target was presented and was selected compared with when it was present but not selected, i.e., when a target was selected it was done so more quickly then when one was not selected [opt-out selected average was $639 \mathrm{~ms}$ (s.d. $147 \mathrm{~ms}$ ) while target selected with opt-out present was $459 \mathrm{~ms}$ (s.d. $54 \mathrm{~ms}$ ); $t(18)=-5.13, p<0.001)]$. 
In contrast saccade trajectory (Figure 3d) was not found to significantly vary due to the presence of the opt-out target $(F(1,18)=2.459, p=0.134)$ but there was a significant main effect of motion coherence $(F(4,72)=7.742, p<0.001)$. There was no significant interaction between the two variables $(F(4,72)=1.350, p=0.260)$. Thus saccade trajectory deviates away from the non-selected target more as motion coherence levels increase but does not differ depending upon the certainty and confidence in the choice.

\section{Discussion}

Participants' performance improved as the motion coherence levels became higher, and saccade latency decreased correspondingly. As motion coherence increased, the saccade path deviated further away from the non-selected target. This replicates the results found by McSorley and McCloy (2009) and Experiment 1, and supports earlier conclusions that the increase in evidence leads to an increase in inhibition of the non-selected target, which is shown by a rise in the amount of deviation away from the non-selected target.

Opt-out target choices were also affected by the level of motion coherence; participants made fewer saccades to the opt-out target when there were higher levels of evidence in the RDK. This is analogous to the findings from the previous experiment, which found that confidence increased as a function of motion coherence. However, saccade trajectory deviations were not significantly different from each other when an opt-out target was present but ignored, compared with those elicited when there was no opt-out target. This suggests that whether or not the opt-out target was present had no effect on the level of inhibition of the non- 
selected target. This again suggests that saccade control and confidence are not based on the same neural activity at the time of the decision.

It was noted that there were not many trials on which participants elected to opt-out of the choice however this was sufficient to produce a behavioural impact. It can be argued that as there was no consequence (either a reward or punishment) for target choice there was little incentive for participants to elect to opt-out of the choice. While we did find that the opt-out target indicated those trials on which confidence was lower, a stronger opt-out target that introduces consequences for target choice (i.e., one that included a monetary reward) may also increase the rate of opting out. It may be that the current opt-out target underestimates the rate of low confidence trials or only captures the extremely low confidence trials. Furthermore, this formulation of the opt-out target would be more in keeping with Kiani and Shadlen (2009) in which the opt-out target was associated with liquid reward.

In the next experiment, we examine whether an induced feeling of confidence, i.e., over confidence or under confidence, in performance directly affects saccade control. If it is the case that confidence judgement and saccade control are derived from the same underlying activity then it may be the case that different levels of preexisting confidence may change this underlying activity, possibly through top down biases and feedback loops, and influence saccade control. One reason to suspect that this might be the case is that being more certain of the location of a saccade target, which could be interpreted as has having greater confidence, has been shown to increase saccade latency and shift saccade trajectory deviation away from distractors (Dorris and Munoz 1998; Kim and Basso 2008; Walker et al. 2006). Thus being more confident in target choice may reduce saccade latencies and increase trajectory deviations away from the non-selected target by increasing the extent of inhibition of the target or the speed by which that inhibition develops. In order to 
examine the impact of confidence on saccade deviation in target choice, we provided manipulated feedback to two groups of participants regarding their performance on the same RDK task as in Experiment 1. This instilled differing levels of confidence between the two groups independent of the external evidence. In a subsequent block of trials, with unmodified feedback appropriate to their choices, we examined whether the differences in confidence felt by the two groups impacted on the same underlying activity from which saccade trajectory control is derived or whether saccade trajectory reflected the evidence supporting target selection to the same extent regardless of confidence. Indeed we find that despite differences in confidence, saccade trajectory deviations were found to be similarly affected by increasing evidence supporting the decision suggesting that confidence is based on post-decisional processing, unlike the saccade trajectory that is based on neural activity at the time of the decision.

\section{Experiment 3}

\section{Method}

Observers

40 observers participated in the experiment, all of whom were naïve undergraduates. Participants were aged between 18 and 25 years old. All had normal, or corrected to normal eyesight. Local ethical approval was obtained, and the study was conducted in accordance with the standards described in the 1964 Declaration of Helsinki. 


\section{Apparatus}

See Experiment 1 for details.

\section{Stimuli}

The motion coherence levels used were 4, 8, 16 and $32 \%$, with forty trials per motion coherence level (twenty in each block), with 160 trials in total. These levels are the same as those employed in Experiment 1.The highest level (64\%) was removed as this may have led to participants becoming more aware of the manipulated feedback.

\section{Design and Procedure}

The design and procedure was the same as Experiment 1 except that feedback was given in the form of the word 'incorrect' or 'correct' appearing in the centre of the screen. There were two blocks of 80 trials; in the first block participants were given manipulated feedback, having been assigned into one of two conditions. Half of the participants were assigned to the high feedback condition that meant that these participants were told they had made the correct decision, regardless of the response they had actually made, $70 \%$ of the time. The remaining participants were assigned to the low condition in which they were told that they had made the incorrect decision $70 \%$ of the time. In the second block of 80 trials, feedback reflected the participant's choice and whether it was correct or not. After being presented with feedback, participants recorded how confident they were in their decision by drawing a mark on a visual analogue scale. It was stressed to the participants that the confidence measurement should reflect how confident they had felt in their decision, even if the feedback had subsequently told them they were wrong. Also, if they felt that they genuinely had no idea about the direction of motion 
that they should guess, and that it was acceptable to put a mark on the 'Low confidence' end of the scale. Participants were required to use the button press to start each trial to drift correct for any movement that occurred when they were recording their confidence. This ensured that they were fixating within one degree visual angle of the fixation cross when the trial started. There were 160 trials in total, which took participants about forty minutes to complete, including practice and setup time.

\section{Data analysis}

See Experiment 1 for details. The same exclusion criteria applied as in Experiment 1, which led to 337 trials (5.3\% of the total number of all trials across all participants) being excluded.

\section{Results}

\section{Feedback manipulation}

The primary aim of manipulating feedback was to modify participants' confidence in their decision independent of the external evidence that supports it. Figure 4 (upper row) shows the normalized confidence ratings (to take into account individual's minimum and maximum score) for both the modified feedback and the unmodified feedback blocks as a function of motion coherence and its development across the experimental block (Figure 4 lower row). 
The feedback manipulation showed a similar effect on confidence across motion coherence levels in both blocks (Figure 4 Upper row). Two separate two-way ANOVAs for each block with feedback and motion coherence as factors showed that motion coherence level did not have a significant impact on confidence ratings in the modified; $(F(3,114)=0.669, p=0.573)$ or the unmodified blocks $(F(3,114)=2.025$, $\mathrm{p}=0.114)$. There was no interaction between the block and the motion coherence level in the modified; $(F(3,114)=1.343, p=0.264)$, or unmodified block; $F(3,114)=0.930, p=0.429)$. There was only a main effect of feedback for both the modified; $(F(1,38)=22.503, p<0.001)$, and unmodified blocks; $(F(1,38)=5.474$, $\mathrm{p}=0.025$ ) with the confidence score for those in the high condition being significantly higher than in the low.

Figure 4 (lower row) shows standardised confidence ratings as a function of trial number (quartile). When the data is examined across the first, modified block participants given more positive feedback about their performance (high condition) became increasingly more confident whereas those given more negative feedback (low condition) became less confident. A two way ANOVA with feedback (high vs low) and quartile as factors supported this interpretation showing, in the modified block, a main effect of feedback $(F(1,38)=15.328, p<0.001)$, no effect of quartile $(F(3,114)=0.255, p=0.858)$ but with an interaction between feedback and quartile $(F(3,114)=3.092, p=0.030)$.

In the unmodified block, confidence did not vary as the block progressed, with those in the high group having higher ratings of confidence throughout. A two way ANOVA on the unmodified feedback block showed only a main effect of feedback $(F(1,38)=5.340, p=0.026)$. The effect of this manipulation was found to continue throughout the unmodified feedback block showing no change as the block 
progressed $(F(3,114)=0.689, p=0.560)$. There was no significant interaction between the quartile and the block; $(F(3,114)=0.746, p=0.527)$. This analysis shows that the confidence manipulation was successful in instilling different levels of confidence in the two groups of participants.

This analysis shows that the feedback manipulation has a dramatic effect of confidence: positive feedback regardless of the level of evidence supporting the decision increases confidence in that decision while negative feedback decreases confidence. This develops over time and is felt across all levels of external evidence. Participants can be safely grouped into those having high confidence and those having low confidence. We now examine the impact of these different internal confidence levels on the performance and saccade control in the unmodified block.

\section{Effect of feedback manipulation on saccade control}

\section{FIGURE 5 HERE}

In order to examine the effect feedback manipulation in the modified block had upon the performance and saccade responses in the unmodified block the data from the unmodified block alone is reported. Figure 5 shows that (a) participants' performance improved as motion coherence increased but (b) saccade latency showed no change. A two-way mixed-factor ANOVA, comparing the two unmodified blocks in which correct feedback was given (high vs low), confirmed that performance showed a significant increase (Motion Coherence; $F(3,114)=11.180, p<0.001$, with a significant linear trend $F(1,38)=13.548, p=0.001$ ) This is largely driven by an improvement in performance in the $32 \%$ motion coherence condition. No interactions $(F(3,114)=0.338, p=0.798)$ were found, but there was a trend for better performance 
for those in the low condition in the unmodified block compared to the high condition $F(1,38)=3.504, p=0.069)$. Saccade latency, on the other hand, showed no difference either as a function of feedback manipulation $(F(1,38)=0.003, p=0.953)$ or motion coherence $(F(3,114)-1.001, p=0.395)^{2}$.

Saccade trajectories (Figure $5 c$ ) tended to deviate further from the non-selected target as levels of motion coherence became higher $(F(3,114)=4.304, p=0.006$; with a significant linear trend $F(1,38)=6.411, p=0.016)$. However, there were no effects of feedback condition $(F(1,38)=0.168, p=0.684)$ and no significant interaction $(F(3,114)=1.437, p=0.236)$.

\section{Discussion}

Previous experiments in this series have suggested a dissociation between the way that evidence accumulated to form a perceptual target choice is used in saccade control and to derive confidence: with saccade metrics reflecting the evidence available to form a target choice and confidence being related to this but subject to additional post-decisional processing. Experiment 3 directly examined whether confidence can be dissociated from the evidence supporting target choice and

\footnotetext{
${ }^{2}$ Unlike in Experiment 1, and McSorley and McCloy (2009), latency was not found to decrease as motion coherence increased. We suggest this to be as a result of the confidence manipulation, and possibly due to the fact that both of these previous experiments included a higher motion coherence level (64\%) that was not present in this experiment. Removing this level, which gives the most evidence for one of the options, thereby making the decision easier, may have made the usual decrease in saccade latency less evident.
} 
whether pre-existing confidence can bias the underlying neural activity that represents the evidence used to select a target and thereby influence saccade control. Participants received manipulated feedback regarding their ability to perform the RDK task, designed to instil a sense of high or low confidence. Those in the high confidence condition were found to have significantly higher confidence ratings than those in the low confidence condition; both in the block when they received manipulated feedback and the second block where accurate task performance feedback was given. Participants' eye movement metrics were then compared in order to ascertain whether this difference in confidence impacted on target choice, i.e., was the non-selected target inhibited more strongly for those with high confidence compared with those with low confidence despite the evidence for the target being the same. It was found that, despite clear differences in confidence, saccade trajectory deviations did not differ showing that confidence is dissociable from, and does not bias the evidence used in the saccade target choice. This suggests that confidence is a function of additional processing over and above the evidence used to make the decision and that confidence in a decision does not feedback on or bias, in some kind of top-down fashion, the underlying activity that represents the evidence used to make these kinds of saccade choices.

\section{General Discussion}

This series of experiments aimed to investigate whether confidence ratings and eye movement responses are linked to the external evidence supporting a decision (i.e., the level of motion coherence within an RDK). Three strands of evidence motivated this. First, a series of single neuron recording experiments have found that higher levels of motion coherence lead to increased neural firing in the LIP (Kiani and 
Shadlen 2009; Roitman and Shadlen 2002; Shadlen and Newsome 2001; Shadlen and Newsome 1996), frontal eye fields (Kim and Shadlen 1999), and the superior colliculus (Horwitz and Newsome 1999). Second, McSorley and McCloy (2009) showed that the evidence supporting target choice is also reflected in deviations of saccade metrics: The saccade deviates away from the non-selected target reflecting the inhibition of that choice. The extent of this deviation increases as there is more evidence for the target choice, reflecting greater inhibition of the non-selected target. Third, it has been reported that the evidence underlying the formation of target choice and confidence in that choice can be derived from this same neural activity (Kiani and Shadlen 2009). In this paper we aimed to establish whether the level of confidence in a decision, and the metrics of the saccade indicating the decision similarly reflect the evidence presented within an RDK, and by extension, the level of neural firing representing the evidence accumulated for that decision. Experiment 1 showed that while confidence and inhibition of the non-selected target increased as evidence supporting the choice increased they did not correlate with each other, suggesting that they do not have a common locus. Experiment 2 and Experiment 3 showed that greater confidence in target choice, shown by not electing to take an opt-out option in Experiment 2 and by directly manipulating confidence in Experiment 3 , did not lead to differences in the inhibition of the non-selected target. Overall, we have found that while confidence and saccade trajectory deviation are related to the formation of target choice they do not seem related to each other. We suggest that saccade control and confidence reflect the evidence for the target choice but confidence may also be subject to further post-decisional processing after target choice formation. It is important to note that what have reported here is not as simple as a null result. Each measure (saccade deviation and confidence rating) clearly 
does change as function of the MC, i.e. the underlying evidence used to support the choice of motion direction impacts on confidence ratings and saccade deviation. The results simply suggest that they do so independently.

Models of confidence judgements are often classified on basis of whether or not the feeling of confidence is derived directly from the evidence that supports the decision. Decisional locus models state that confidence in the decision can be read out directly from the accumulated evidence at the time the decision was made (as per Kiani and Shadlen 2009; Kepecs et al. 2008), whereas post-decisional locus models state that confidence is based on information that arrives after the decision (such as Pleskac and Busemeyer, 2010). Our results are consistent with such post-decisional accounts.

There are a number of different scenarios in which post-decisional processes may account for our results. One possibility is that confidence ratings are derived from further processing than that which is available to the target choice itself. This may involve the same information as that used to make the decision, but which may be accruing internal noise or simply decaying thereby changing its quality in both cases. On the other hand there may be differences in information that is available to choice and confidence. One may have access to information not available to the other. This may take the form of a dual-channel model in which one channel supports conscious processing while the other unconscious processing (Del Cul et al. 2009; Morewedge and Kahneman 2010) or other more complex asymmetries in information access (e.g. Pasquali et al. 2010). In the context of our experiments, confidence may be based on conscious processing and the target choice may be based on unconscious 
processing. A further possibility is that confidence and target choice are based on evidence that has been differently transformed giving different representations.

In summary, this series of experiments replicate the effects shown by McSorley and McCloy (2009); as motion coherence increased, saccade latency decreased, and correct performance and trajectory deviation away from the non-selected target increased. This innovative way of looking at eye movement metrics was extended in this paper to investigate confidence in a perceptual decision. We were specifically interested in whether it was based on the same neural firing, caused by the evidence within the RDK, as the saccade metrics or on further processing. Our results suggest that confidence ratings are based on further processing after the decision has been made. 


\section{References}

Audley RJ (1964) Decision making. British Medical Bulletin 20:27-31.

Britten KH, Newsome WT, Shadlen MN, et al. (1996) A relationship between behavioral choice and the visual responses of neurons in macaque MT. Vis Neurosci 13:87-100.

Del Cul A, Dehaene S, Reyes P, et al. (2009) Causal role of prefrontal cortex in the threshold for access to consciousness. Brain 132:2531-2540. doi: 10.1093/brain/awp111

Dorris MC, Munoz DP (1998) Saccadic probability influences motor preparation signals and time to saccadic initiation. J Neurosci 18:7015-7026.

Findlay J, Brown V (2006) Eye scanning of multi-element displays: II. Saccade planning. Vision Res 46:216-227.

Findlay J, Walker R (1999) A model of saccade generation based on parallel processing and competitive inhibition. Behav Brain Sci 22:661-674.

Gold JI, Shadlen MN (2000) Representation of a perceptual decision in developing oculomotor commands. Nature 404:390-394. doi: 10.1038/35006062

Gold JI, Shadlen MN (2001) Neural computations that underlie decisions about sensory stimuli. Trends Cogn Sci 5:10-16.

Gold JI, Shadlen MN (2002) Banburismus and the brain: decoding the relationship between sensory stimuli, decisions, and reward. Neuron 36:299-308.

Harvey N (1997) Confidence in judgment. Trends Cogn Sci 1:78-82. 
Horwitz GD, Newsome WT (1999) Separate Signals for Target Selection and

Movement Specification in the Superior Colliculus. Science 284:1158-1161. doi: 10.1126/science.284.5417.1158

Horwitz GD, Newsome WT (2001) Target selection for saccadic eye movements: prelude activity in the superior colliculus during a direction-discrimination task. $J$ Neurophysiol 86:2543-2558.

Juslin P, Olsson H (1997) Thurstonian and Brunswikian origins of uncertainty in judgment: a sampling model of confidence in sensory discrimination. Psychol Rev 104:344-366.

Kepecs A, Uchida N, Zariwala HA, Mainen ZF (2008) Neural correlates, computation and behavioural impact of decision confidence. Nature 455:227-231. doi: $10.1038 /$ nature07200

Kiani R, Shadlen MN (2009) Representation of Confidence Associated with a Decision by Neurons in the Parietal Cortex. Science 324:759-764. doi: 10.1126/science. 1169405

Kim B, Basso MA (2008) Saccade Target Selection in the Superior Colliculus: A Signal Detection Theory Approach. J Neurosci 28:2991-3007. doi: 10.1523/JNEUROSCI.5424-07.2008

Kim JN, Shadlen MN (1999) Neural correlates of a decision in the dorsolateral prefrontal cortex of the macaque. Nat Neurosci 2:176-185. doi: 10.1038/5739

Laming DRJ (1968) Information theory of choice-reaction times. Academic Press, New York 
Link S (1992) The Wave Theory of Difference and Similarity. Lawrence Erlbaum Associates, New Jersey

Link S, Heath R (1975) A sequential theory of psychological discrimination. Psychometrika 40:77-105. doi: 10.1007/BF02291481

Ludwig CJH, Gilchrist ID (2002) Measuring saccade curvature: a curve-fitting approach. Behav Res Methods Instrum Comput 34:618-624.

Masson M, Loftus G (2003) Using confidence intervals for graphically based data interpretation. Can J Exp Psychol 57:203.

McSorley E, Findlay JM (2003) Saccade target selection in visual search: accuracy improves when more distractors are present. J Vis 3:877-892.

McSorley E, Cruickshank AG, Inman LA (2009) The development of the spatial extent of oculomotor inhibition. Brain Res 1298:92-98.

McSorley E, McCloy R (2009) Saccadic eye movements as an index of perceptual decision-making. Exp Brain Res 198:513-520.

McSorley E, McCloy R, Lyne C (2012) The spatial impact of visual distractors on saccade latency. Vision Res 60:61-72. doi: 10.1016/j.visres.2012.03.007

Meeter M, Van der Stigchel S, Theeuwes J (2010) A competitive integration model of exogenous and endogenous eye movements. Biol Cybern 102:271-291.

Morewedge CK, Kahneman D (2010) Associative processes in intuitive judgment. Trends Cogn Sci 14:435-440. doi: 10.1016/j.tics.2010.07.004

Ottes FP, Van Gisbergen JA, Eggermont JJ (1985) Latency dependence of colour- 
based target vs nontarget discrimination by the saccadic system. Vision Res 25:849-862.

Pasquali A, Timmermans B, Cleeremans A (2010) Know thyself: metacognitive networks and measures of consciousness. Cognition 117:182-190. doi: 10.1016/j.cognition.2010.08.010

Petrusic WM, Baranski JV (2003) Judging confidence influences decision processing in comparative judgments. Psychon Bull Rev 10:177-183.

Pleskac TJ, Busemeyer JR (2010) Two-stage dynamic signal detection: a theory of choice, decision time, and confidence. Psychol Rev 117:864-901. doi: $10.1037 / \mathrm{a} 0019737$

Ratcliff R (1978) A theory of memory retrieval. Psychol Rev 85:59.

Roitman JD, Shadlen MN (2002) Response of neurons in the lateral intraparietal area during a combined visual discrimination reaction time task. J Neurosci 22:9475-9489.

Shadlen MN, Newsome WT (2001) Neural basis of a perceptual decision in the parietal cortex (area LIP) of the rhesus monkey. J Neurophysiol 86:1916-1936.

Shadlen MN, Newsome WT (1996) Motion Perception: Seeing and Deciding. Proc Natl Acad Sci USA 93:628-633.

Stone M (1960) Models for choice-reaction time. Psychometrika 25:251-260.

Tipper SP, Howard LA, Paul MA (2001) Reaching affects saccade trajectories. Exp Brain Res 136:241-249. 
Van der Stigchel S, Meeter M, Theeuwes J (2006) Eye movement trajectories and what they tell us. Neurosci Biobehav Rev 30:667-679. doi:10.1016/j.neubiorev.2005.12.001

Vickers D (1979) Decision Processes in Visual Perception. Academic Press, New York.

Walker R, Deubel H, Schneider WX, Findlay JM (1997) Effect of remote distractors on saccade programming: evidence for an extended fixation zone. $\mathrm{J}$ Neurophysiol 78:1108-1119.

Walker R, McSorley E, Haggard P (2006) The control of saccade trajectories: direction of curvature depends on prior knowledge of target location and saccade latency. Percept Psychophys 68:129-138.

Yeung N, Summerfield C (2012) Metacognition in human decision-making: confidence and error monitoring. Philos T Roy Soc B 367:1310-1321. doi: 10.1073/pnas.1104517108 


\section{Figure Legends}

Fig. 1 Saccade decision task. After a random fore period of 800-1200 ms, the targets appeared, followed in quick succession by the RDK stimulus. The RDK was present for $125 \mathrm{~ms}$, and the targets remained for a further $875 \mathrm{~ms}$. The direction of the RDK stimulus here is represented by arrows (not present in the display). For clarity fewer dots are shown here than were actually drawn on any one video frame. The upper right panel of the figure shows a breakdown of saccade analysis. Each saccade was deemed correct if it fell within 45 deg of the target of dot motion (shown here as dashed lines bracketing each target). A number of eye movement responses from one participant are shown for the left target. For the right a schematic eye trace is shown. On this $\alpha$ shows the trajectory deviation from a direct, straight, saccade and $\beta$ shows the landing position deviation from the centre of the target.

Fig. 2 Performance measures. The percentage of correct responses (a) rose as motion coherence of the RDK increased, while saccade latency (b) decreased. Both the feeling of confidence in the choice (c) and saccade trajectory deviation away from non-selected target (d) were also found to increase with motion coherence. Error bars are repeated measures error bars (Masson and Loftus 2003)

Fig. 3 Percentage of correct responses (a) increased when the opt-out target was present (diamonds) and when it was not (triangles). This was found to be stronger when the opt-out target was present. (b) Percentage of opt-out target choice decreased as motion coherence increased. (c) Saccade latencies were found to 
decrease across the motion coherence levels. (d) Saccade trajectory deviations were not different when the opt-out target was (diamonds) and was not (triangles) present but overall deviations were away from the non-selected target and that this increased with increasing motion coherence. Error bars are repeated measures error bars (Masson \& Loftus, 2003)

Fig. 4 Upper row: Normalised confidence ratings for both the modified feedback and the unmodified feedback blocks across motion coherence levels. Diamonds show results from the high performance feedback manipulations and triangles the low performance feedback condition. Lower row: Normalized confidence ratings for both the modified feedback and the unmodified feedback blocks as a function of trial number (quartile). Blocks have been quartiled running from the first $25 \%$ of trials to the last $25 \%$. Error bars show the between subjects standard error of mean

Fig. 5 The number of correct responses (a) saccade latency (b) and saccade trajectory deviations (c) as a function of motion coherence for the high and low confidence conditions. Error bars show the between subjects standard error of mean 
FIGURE 1

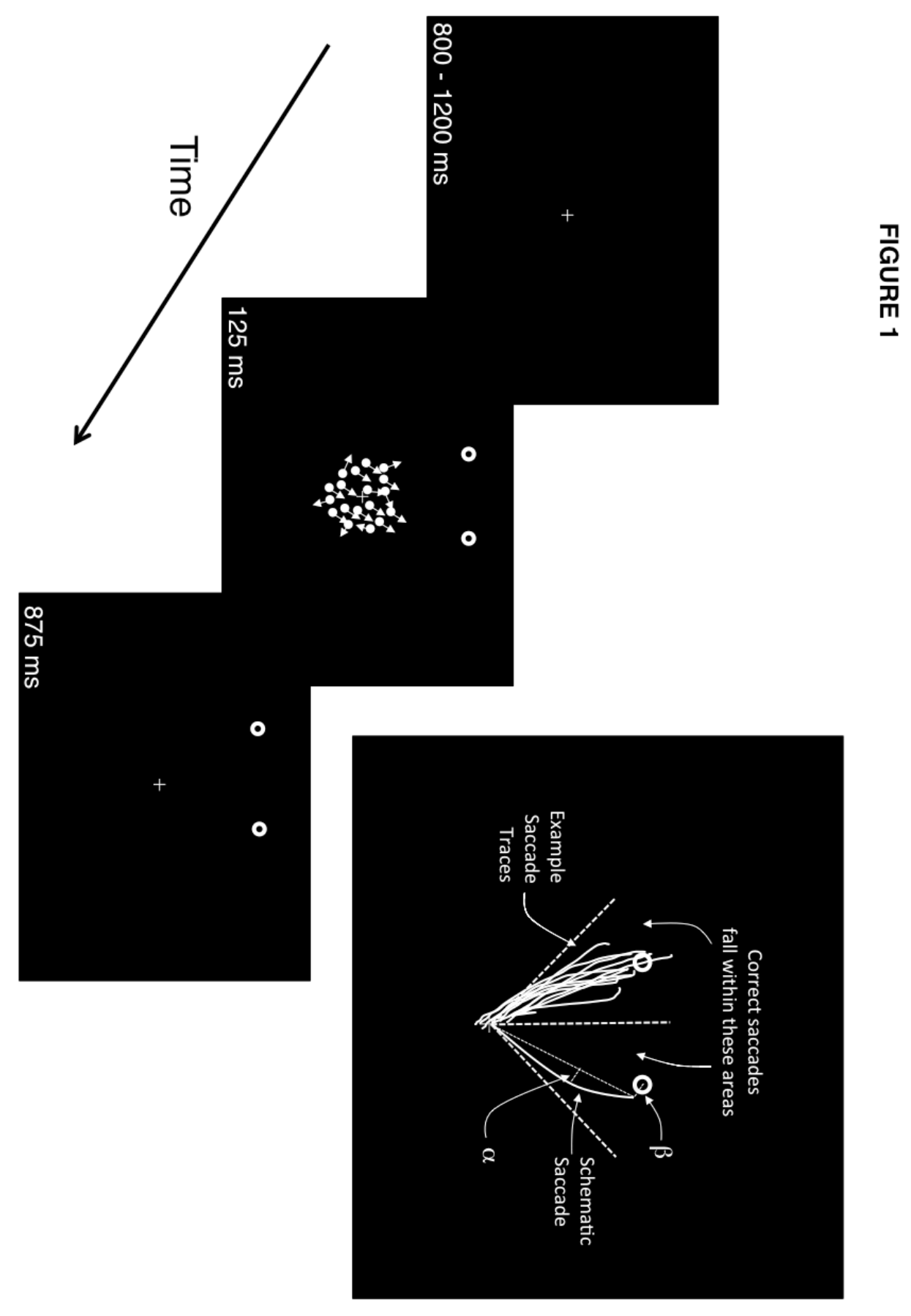


FIGURE 2
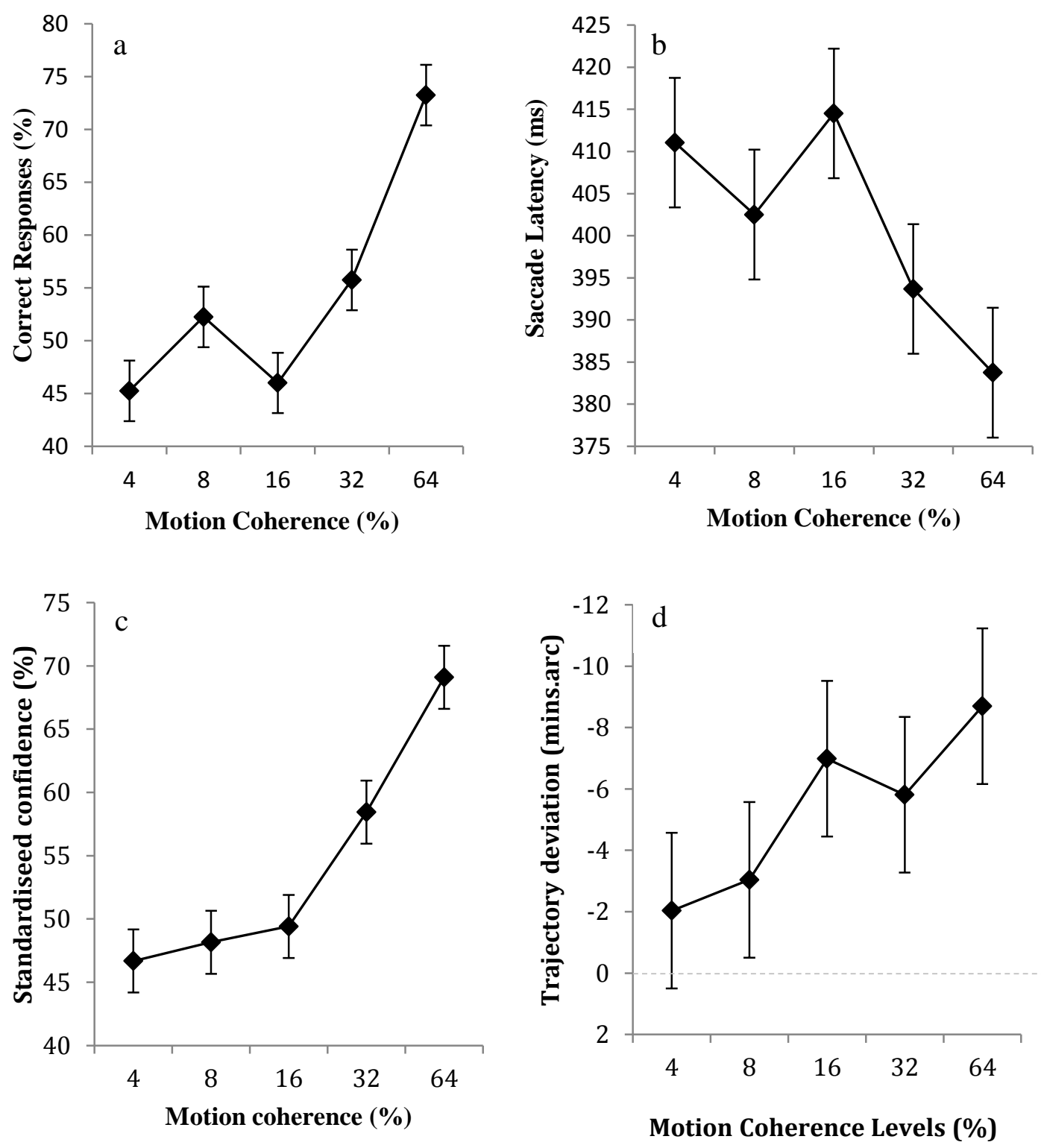


\section{FIGURE 3}
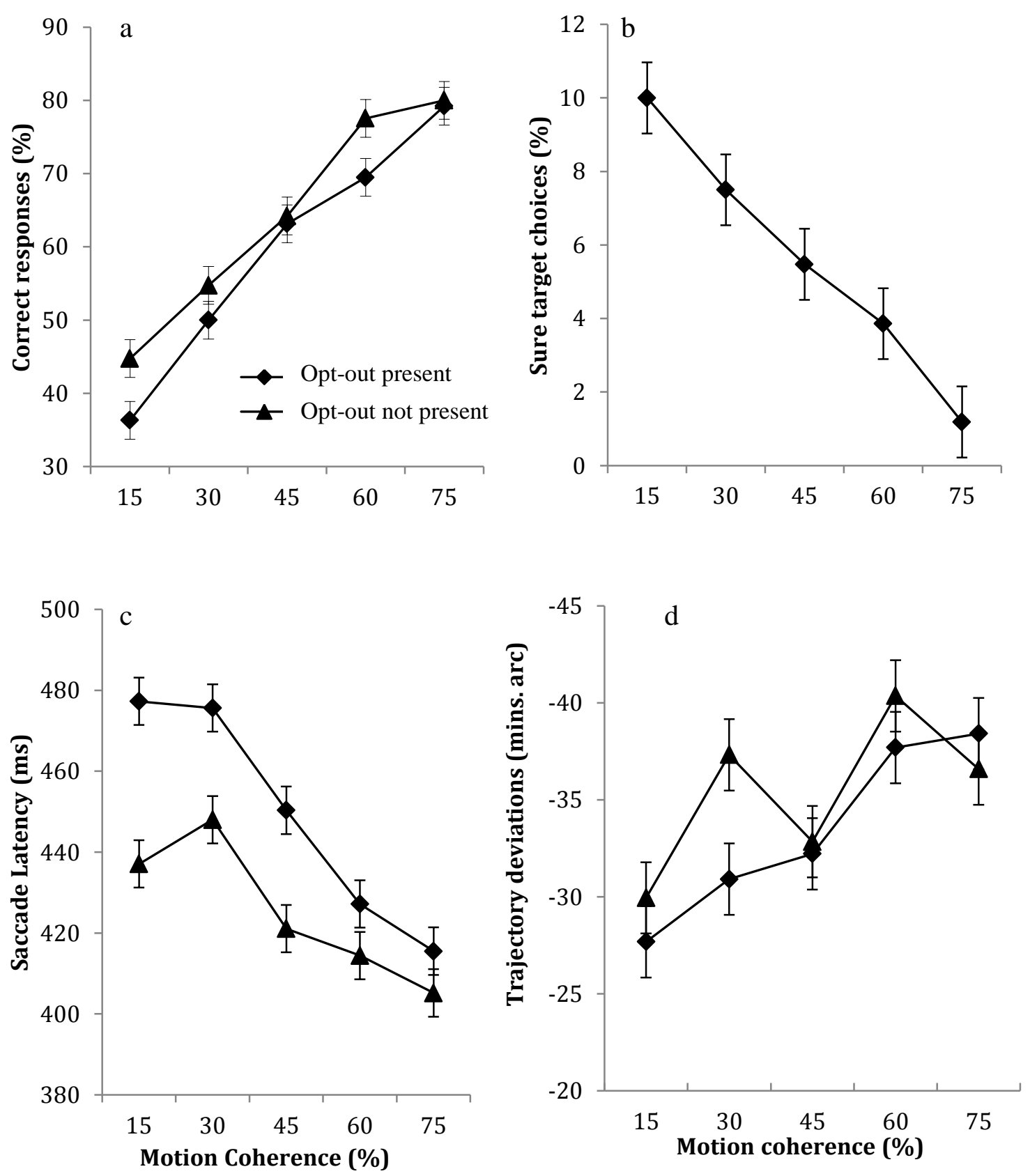
FIGURE 4
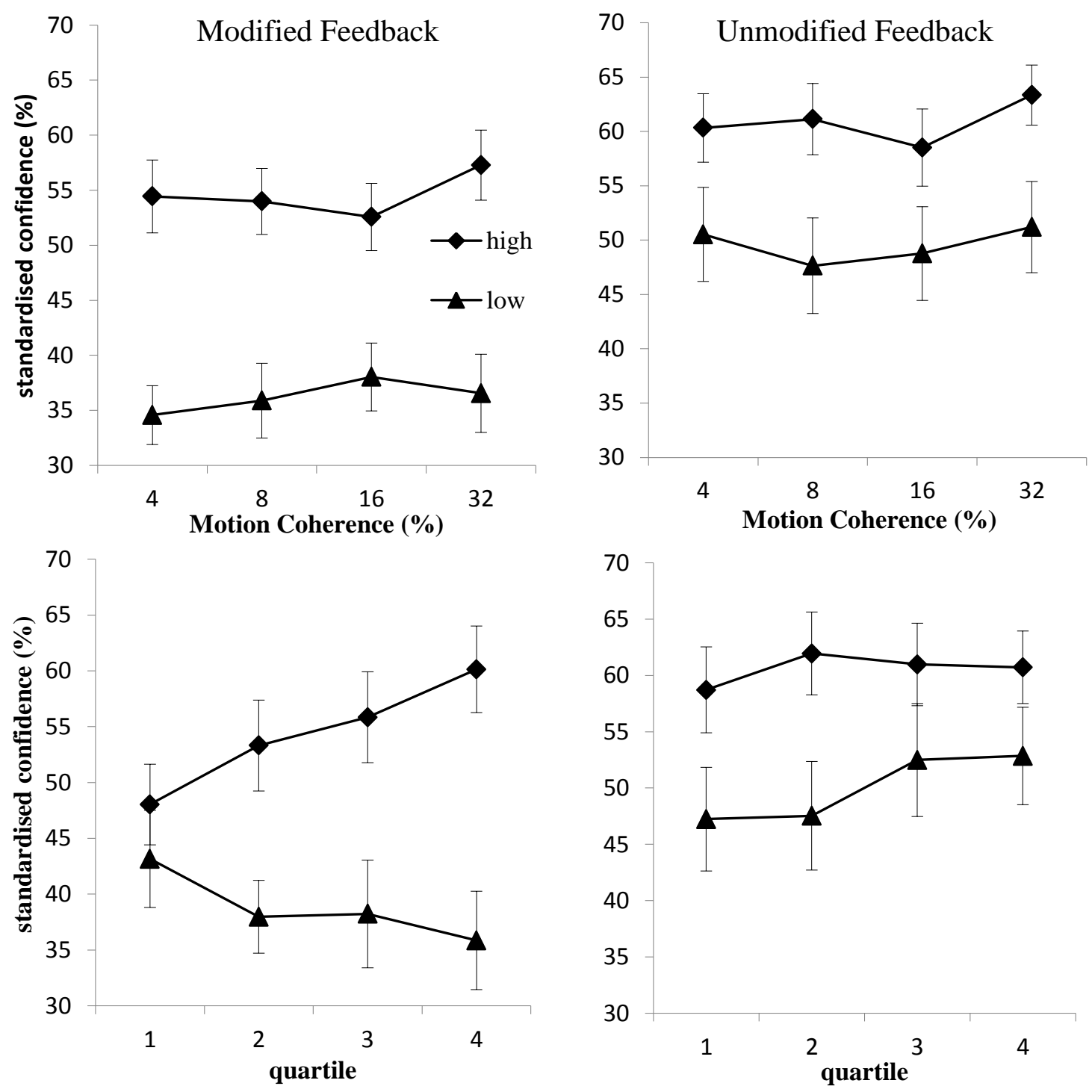


\section{FIGURE 5}
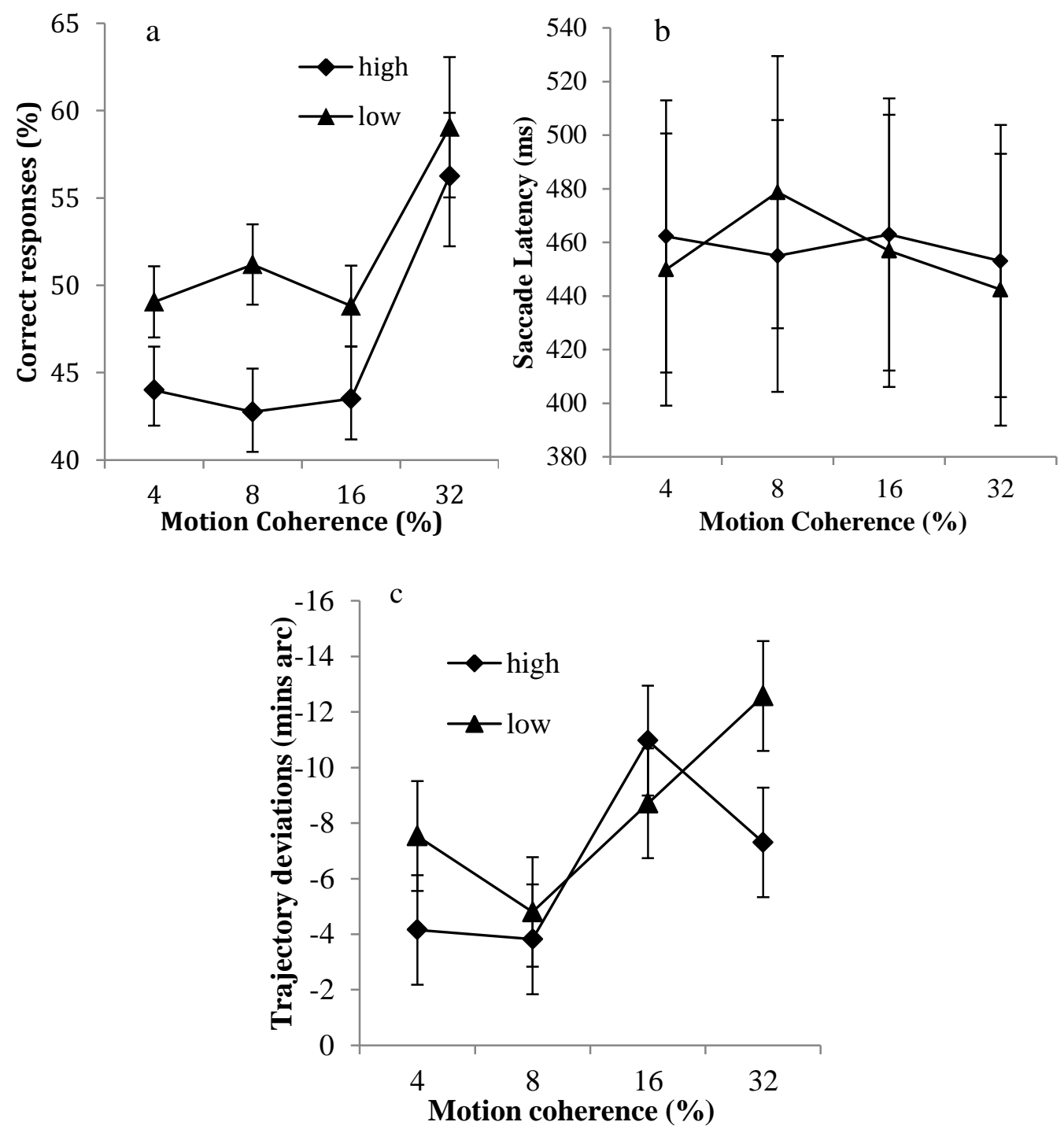